\title{
Ectopic Expression of DNA Repair Enzymes Modulates Survival following Ultraviolet Irradiation Challenge
}

Stuti P. Garg ${ }^{1}$, Irina G. Minko¹, Erdem Coskun²,3, Onur Erdem²,4, Pawel Jaruga², Miral Dizdaroglu², R. Stephen Lloyd ${ }^{1,5,6, *}$

${ }^{1}$ Oregon Institute of Occupational Health Sciences, Oregon Health \& Science University

Portland, Oregon 97239-3098

2 Biomolecular Measurement Division, National Institute of Standards and Technology, Gaithersburg MD 20899-8315

${ }^{3}$ Department of Toxicology, Faculty of Pharmacy, Gazi University, Ankara, Turkey

4 Department of Pharmaceutical Toxicology, University of Health Sciences, 06010 Ankara, Turkey

${ }^{5}$ Department of Molecular and Medical Genetics, Oregon Health \& Science University Portland, Oregon 97239-3098

${ }^{6}$ Department of Physiology and Pharmacology, Oregon Health \& Science University Portland, Oregon 97239-3098

* Corresponding author; 3181 SW Sam Jackson Park Rd. Portland, OR 97239 503-494-9957 (voice)

503-494-6831 (fax)

lloydst@ohsu.edu

Key words: cyclobutane pyrimidine dimers, 6-4 photoproducts, UVB irradiation, solar-simulated light, Chlorella virus pyrimidine dimer glycosylase, UVDE, formamidopyrimidines 


\section{Abstract}

In Escherichia coli, the nucleotide excision repair (NER) pathway removes ultraviolet (UV) lightinduced cyclobutane pyrimidine dimers (CPDs) and 6-4 dipyrimidine photoproducts (6-4 PPs). Activation of alternative repair pathways, such as base excision repair (BER) and nucleotide incision repair (NIR), is inoperative because this organism lacks both the necessary BER DNA glycosylase and NIR UV endonuclease to initiate repair of these lesions. To determine if initiation of either pathway would enhance survival to biologically-relevant UV irradiation, the BER and NIR pathways were activated by expression of Chlorella virus-1 pyrimidine dimer glycosylase (cv-pdg) and Schizosaccharomyces pombe UV endonuclease (UVDE), respectively. The substrate specificity of cv-pdg includes CPDs and ring-fragmented purines, 4,6-diamino-5-formamidopyrimidine and 2,6-diamino-4-hydroxy-5-formamidopyrimidine, but not 6-4 PPs. In contrast, while UVDE incises DNA containing CPDs and 6-4 PPs, it was not previously known if the substrate specificity of UVDE included DNA containing ring-fragmented purines. Mass spectrometry was used to establish that these oxidatively-induced lesions were not substrates for UVDE. Expression of either cV-pdg or UVDE in NER-deficient E. coli significantly enhanced survival following UVB irradiation, but not to the levels of wild type (WT) cells. Survival of NER-proficient, homologous recombination-deficient cells could also be significantly enhanced by expression of either enzyme, suggesting that in response to UVB exposure, interactions between NER and activated BER or NIR pathways could be additive. Further, expression of cv-pdg or UVDE in WT E. coli enhanced survival following solarsimulated light (SSL) exposures. 


\section{Introduction}

The spectrum of ultraviolet (UV) irradiation can be divided into three major regions based on differences in photochemistry and biological interactions. UVC irradiation $(<280 \mathrm{~nm})$ is strongly absorbed by DNA, producing cyclobutane pyrimidine dimers (CPDs), 6-4 photoproducts (6-4 PPs), and many other DNA lesions. However, these wavelengths are not germane to UV exposures at the Earth's surface due to atmospheric attenuation. UVB (280-315 nm) is the most biologically damaging component of sunlight that reaches the Earth's surface. Although it accounts for $<5 \%$ of the UV that passes through the ozone, the UVB causes direct photochemical DNA damage, including CPDs and 6-4 PPs [1]. The role of these lesions in UVinduced mutagenesis has been established, particularly in human clinical samples of skin tumors and pre-cancerous lesions [2]. In addition, UVB has been demonstrated to oxidatively induce DNA damage, both directly and via reactive oxygen species [3,4]. Pure DNA only weakly absorbs UVA (315-400 nm), but in cells, endogenous photosensitizers amplify the DNA damaging effects of these wavelengths, resulting in the formation of the same DNA lesions induced following exposure to shorter wavelengths $[1,4,5]$. UVA has been implicated in photoaging, immunosuppression, and the generation of reactive oxygen species [3,6-8].

In Escherichia coli (E. coli), DNA repair of CPDs and 6-4 PPs primarily proceeds via the Nucleotide Excision Repair (NER) pathway, with the initiation of this pathway requiring the coordinated activities of the UvrA, UvrB, and UvrC proteins. Defects in any of these proteins confer significant increases in UV-induced cytotoxicity [9,10]. Additionally, CPDs can undergo photoreversal under appropriate conditions through the activity of photolyase [10]. The homologous recombination (HR) and translesion DNA synthesis pathways that constitute damage tolerance mechanisms rather than repair processes, are also important for cellular survival following UV exposure [11].

While not present in E. coli, many bacteria and viruses utilize DNA glycosylases to initiate repair of CPDs via the Base Excision Repair (BER) pathway $[12,13]$. These enzymes cleave the 
$\mathrm{N}$-glycosidic bond of the 5' pyrimidine of CPD and subsequently incise the phosphodiester backbone at the abasic site via a lyase mechanism [14-16]. Several bacteriophage species of the Myoviridae and Phycodnaviridae (Chlorella virus) families possess such pyrimidine dimer glycosylases (pdgs) [17-20], and similar sequences have been found in bacterial genomes, only distantly related to each other (Brucella, Prochlorococcus, Bordetella, Haemophilus, Pasteurella) [21-23]. The best characterized of the viral enzymes are the T4 and Paramecium bursari chlorella virus-1 pdgs (T4-pdg and cv-pdg, respectively) [24-27]. In addition to CPDs, cVpdg has been reported to catalyze incision of a subset of oxidatively-induced DNA lesions, including 4,6-diamino-5-formamidopyrimidine (FapyAde) and 2,6-diamino-4-hydroxy-5formamidopyrimidine (FapyGua) [26]. The formation of these ring-fragmented purines in UVBand UVC-irradiated DNA in aqueous solution and in the skin of UVB-irradiated mice has been demonstrated [28,29]. Since E. coli has all necessary BER components to complete glycosylase-initiated repair, it was hypothesized that complementation with pdgs would enhance survival following UV exposure. Indeed, partial complementation has been demonstrated for UVC exposure of an E. coli strain that was defective in both NER and HR [30-32].

In addition to the use of BER to augment or substitute for $E$. coli NER, the Nucleotide Incision Repair (NIR) pathway for UV-induced photoproducts can be initiated by the activity of a UV damage-specific endonuclease (UVDE) [33]. Schizosaccharomyces pombe UVDE has a broad substrate range that includes CPDs, 6-4 PPs, abasic sites, small loops, and mismatches [34-36]. However, substrate specificity of UVDE regarding oxidatively-induced DNA base damage has not been previously characterized, except for the demonstration of a weak activity on DNA containing a site-specific dihydrouracil paired with non-cognate Gua as opposed to Ade [34]. In contrast to the glycosylase mechanism of pdgs, UVDE is an endonuclease that cleaves the DNA phosphodiester backbone $5^{\prime}$ to the damage site, leaving a $5^{\prime}$ phosphate and a $3^{\prime} \mathrm{OH}$, thus initiating the NIR pathway. 
Although prior biochemical and structural biology investigations have extensively characterized the pdg and UVDE enzymes [24-27,34-36], the biological efficacies of activating either pathway in response to exposures to biologically relevant UV wavelengths have not been performed. Thus, the abilities of cV-pdg and UVDE to alter biological endpoints associated with UVB and solar simulated light (SSL) exposures were examined following expression of these enzymes in E. coli strains that either were wild type (WT) for DNA repair and tolerance pathways or had a deficiency in NER or HR.

\section{Materials and Methods}

\subsection{UVB and SSL sources}

UVB irradiation was performed using an Ultraviolet Products $302 \mathrm{~nm}$ UV lamp (15-watt) without filter. The intensity was determined using an IL1400A meter (International Light INC) with the SEL204/UVB/W detection probe (measurement range: $275-310 \mathrm{~nm}$ ) and the doses were calculated. SSL was produced by a Newport Class ABA 1600 W Solar Simulator, with atmospheric attenuation filter. The intensities of UVA and UVB components were separately measured using SEL033/UVA/TD (measurement range: $315-390 \mathrm{~nm}$ ) and SEL204/UVB/W detection probes. The dose rates for UVA and UVB were $\sim 5.7$ and $0.2 \mathrm{~kJ} / \mathrm{m}^{2} / \mathrm{min}$, respectively, for the fixed-height irradiation. The relative yield of UVA and UVB were $~ 96$ and $4 \%$, respectively. All exposures to SSL were performed under these standard conditions. For simplicity, the data are reported as a function of irradiation time.

\subsection{E. coli strains}

The genotypes of the E. coli strains used in the UVB and SSL analyses are given in Table 1. The parental AB1157 strain and isogenic mutants that were either defective in NER (AB2500, uvrA6) or HR (AB2487, recA13) were obtained from the E. coli Genetic Stock Center (Yale University). The DH5 $\alpha$ and BL21 (DE3) E. coli strains used for cloning and initial characterization of DNA constructs were purchased from Invitrogen.

\subsection{Cell survival following exposure to UVB or SSL}


Following overnight growth in LB media supplemented with ampicillin (100 $\mu \mathrm{g} / \mathrm{mL})$, cells were serially diluted. Equal quantities of multiple dilutions were aliquoted in triplicate onto prewarmed LB-ampicillin plates and the media allowed to absorb into the agar. Following irradiation with either UVB or SSL, plates were incubated at $30^{\circ} \mathrm{C}$ for $24-48 \mathrm{~h}$, until colonies were $\sim 2 \mathrm{~mm}$ in diameter. Only plates containing $>25$ colonies were analyzed. All experiments were repeated a minimum of 3 times after establishing the optimal exposure conditions for each strain and source of irradiation. Data are expressed as means with standard errors. The $p$ values were calculated using two-tailed Student's $t$-test. Values are considered significant for $p \leq 0.05$.

\subsection{Cloning and expression of cV-pdg and UVDE}

The expression construct for cv-pdg was previously described [37]. The gene encoding the catalytically-active, N-terminally truncated form of UVDE $(\Delta 228)$ (gift from Dr. Paul Doetsch, Emory University) was inserted into a pET-22b vector using unique Nde I and Hind III sites, and the sequence of the cloned gene was verified by Sanger sequencing. Plasmid expression vectors were introduced into BL21 (DE3) cells per the supplier's protocol, and crude cellular lysates were prepared from the overnight cultures to assay for steady-state expression of active enzymes. Expression of the enzymes was confirmed by incubation of cellular lysates with UVCirradiated plasmid DNA (data not shown). The pET-22b vectors containing the $c v-p d g$ or uvde( $\Delta 228)$ gene or no insert were introduced by electroporation into AB1157, AB2500, and AB2487 cells. Ampicillin resistant clones were selected and stored as frozen glycerol stocks. Cultures used for cell survival analyses were made from fresh single-colony isolates derived from glycerol stocks.

\subsection{Preparation of DNA samples}

Commercially available calf thymus DNA was dissolved in phosphate buffer $(\mathrm{pH} 7.4)(0.3$ $\mathrm{mg} / \mathrm{mL}$ ) at $4 \stackrel{\circ}{\circ}$. The DNA solution was saturated with $\mathrm{N}_{2} \mathrm{O}$ for $30 \mathrm{~min}$, irradiated with $\gamma$-rays in a ${ }^{60} \mathrm{Co} \gamma$ ray-source at a dose of $10 \mathrm{~Gy}$ (dose rate $5.17 \mathrm{~Gy} / \mathrm{min}$ ), and DNA was dialyzed against water at $4^{\circ} \mathrm{C}$ for $18 \mathrm{~h}$. After dialysis, the DNA concentration was measured by absorption 
spectrophotometry. Aliquots $(50 \mu \mathrm{g})$ of the DNA sample were supplemented with aliquots of the internal standards, i.e., FapyAde- ${ }^{13} \mathrm{C},{ }^{15} \mathrm{~N}_{2}$, FapyGua- ${ }^{13} \mathrm{C},{ }^{15} \mathrm{~N}_{2}, 8-\mathrm{OH}-A d e-{ }^{13} \mathrm{C},{ }^{15} \mathrm{~N}_{2}, 8-\mathrm{OH}-\mathrm{Gua}-$ ${ }^{15} \mathrm{~N}_{5}, 5-\mathrm{OH}-$ Cyt- ${ }^{13} \mathrm{C},{ }^{15} \mathrm{~N}_{2}, 5-\mathrm{OH}-U r a-{ }^{13} \mathrm{C}_{4},{ }^{15} \mathrm{~N}_{2}$, ThyGly- ${ }^{2} \mathrm{H}_{4}, 5-\mathrm{OH}-5-$ MeHyd- ${ }^{13} \mathrm{C},{ }^{15} \mathrm{~N}_{2}$ and 5,6diOH-Ura- ${ }^{13} \mathrm{C},{ }^{15} \mathrm{~N}_{2}$ (isodialuric acid- ${ }^{13} \mathrm{C},{ }^{15} \mathrm{~N}_{2}$ ), which are a part of the NIST Standard Reference Material 2396 Oxidative DNA Damage Mass Spectrometry Standards (for details see http://www.nist.gov/srm/index.cfm and https://wwws.nist.gov/srmors/view_detail.cfm?srm=2396). The samples were dried in a SpeedVac under vacuum and kept at $4{ }^{\circ} \mathrm{C}$ until use.

For the enzymatic treatment of DNA samples, two different buffer solutions were used: Buffer 1 (no divalent metal ions): 50 mM phosphate buffer (pH 7.4), 100 mM KCl, 1 mM EDTA, and $0.1 \mathrm{mM}$ dithiothreitol; Buffer 2 (plus divalent metal ions): $50 \mathrm{mM}$ phosphate buffer (pH 7.4), $100 \mathrm{mM} \mathrm{KCl}, 2 \mathrm{mM} \mathrm{MnCl}$, $2 \mathrm{mM} \mathrm{MgCl}$, $1 \mathrm{mM}$ EDTA, and $0.1 \mathrm{mM}$ dithiothreitol.

DNA samples $(50 \mu \mathrm{g})$ were dissolved in $50 \mu \mathrm{L}$ of an incubation buffer and incubated with 5 $\mu \mathrm{g}$ of UVDE or $5 \mu \mathrm{g} \mathrm{cV}$-pdg for $1 \mathrm{~h}$ at $37^{\circ} \mathrm{C}$. Another set of samples was sequentially incubated with $5 \mu \mathrm{g}$ of UVDE for $1 \mathrm{~h}$ and with $5 \mu \mathrm{g}$ of cv-pdg for an additional $1 \mathrm{~h}$. DNA samples to be used as controls were incubated without any enzyme. Four independently prepared samples were used for each data point. After incubation, $125 \mu \mathrm{L}$ of cold ethanol (kept at $-20{ }^{\circ} \mathrm{C}$ ) were added to the samples to stop the reaction and to precipitate DNA. The samples were kept at $-20^{\circ} \mathrm{C}$ for 1 $\mathrm{h}$, and then centrifuged at $15,000 \mathrm{~g}$ for $30 \mathrm{~min}$ at $4^{\circ} \mathrm{C}$. DNA pellets and supernatant fractions were separated. Ethanol was removed from the supernatant fractions under vacuum in a SpeedVac. Aqueous supernatant fractions were frozen at $-80^{\circ} \mathrm{C}$ for $1 \mathrm{~h}$ and then lyophilized to dryness for $18 \mathrm{~h}$.

\subsection{Measurement of DNA base lesions by gas chromatography-tandem mass spectrometry} (GC-MS/MS)

To each lyophilized supernatant fraction, $60 \mu \mathrm{L}$ of a mixture of nitrogen-bubbled bis(trimethylsilyl)trifluoroacetic acid [containing trimethylchlorosilane (1\%; v/v)] (BSTFA) (Pierce, 
Rockford, III.) and pyridine (Sigma-Aldrich, St. Louis, MO) (1:1, v/v) was added. The samples were vortexed and purged individually with ultra-high-purity nitrogen, tightly sealed with Tefloncoated septa, and then heated at $120^{\circ} \mathrm{C}$ for $30 \mathrm{~min}$. After cooling, the clear samples were removed and placed in vials used for injection onto the GC-column. Vials were purged with ultra-high-purity nitrogen and tightly sealed with Teflon-coated septa. Aliquots $(4 \mu \mathrm{L})$ of derivatized samples were analyzed by GC-MS/MS under the conditions described previously [38]. On the basis of the known mass spectra of trimethylsilyl derivatives of DNA base lesions [39-41], the following mass transitions were used for identification and quantification: $\mathrm{m} / \mathrm{z} 369 \rightarrow$ $m / z 280$ and $m / z 372 \rightarrow m / z 283$ for FapyAde and FapyAde $-{ }^{13} \mathrm{C},{ }^{15} \mathrm{~N}_{2}$ respectively; $m / z 457 \rightarrow$ $m / z 368$ and $m / z 460 \rightarrow m / z 371$ for FapyGua and FapyGua- ${ }^{13} \mathrm{C},{ }^{15} \mathrm{~N}_{2}$, respectively; $m / z 367 \rightarrow$ $m / z 352$ and $m / z 370 \rightarrow m / z 355$ for $8-O H$-Ade and $8-O H$-Ade- ${ }^{13} \mathrm{C},{ }^{15} \mathrm{~N}_{2}$, respectively; $m / z 455 \rightarrow$ $m / z 440$ and $m / z 460 \rightarrow m / z 445$ for $8-O H$-Gua and $8-O H-G u a-{ }^{15} \mathrm{~N}_{5}$, respectively; $m / z 343 \rightarrow$ $m / z 342$, and $m / z 346 \rightarrow m / z 345$ for $5-\mathrm{OH}-$ Cyt and $5-\mathrm{OH}-$ Cyt- ${ }^{13} \mathrm{C},{ }^{15} \mathrm{~N}_{2}$, respectively; $\mathrm{m} / \mathrm{z} 344 \rightarrow$ $m / z 343$, and $m / z 350 \rightarrow m / z 349$ for $5-\mathrm{OH}-U r a$ and $5-\mathrm{OH}-U r a-{ }^{13} \mathrm{C}_{4},{ }^{15} \mathrm{~N}_{2}$, respectively; $m / z 448$ $\rightarrow m / z 259$ and $m / z 452 \rightarrow m / z 262$ for ThyGly and ThyGly- ${ }^{2} \mathrm{H}_{4}$, respectively; $m / z 331 \rightarrow m / z$ 316 and $m / z 334 \rightarrow m / z 319$ for 5-OH-5-MeHyd and 5-OH-5-MeHyd- ${ }^{13} \mathrm{C},{ }^{15} \mathrm{~N}_{2}$, respectively; $m / z$ $432 \rightarrow m / z 417$ and $m / z 435 \rightarrow m / z 420$ for 5,6-diOH-Ura and 5,6-diOH-Ura- ${ }^{13} \mathrm{C},{ }^{15} \mathrm{~N}_{2}$, respectively. The quantification was achieved using integrated areas of the signals of the mass transitions of the monitored DNA base lesions and those of their stable isotope-labeled analogues.

\section{Results and Discussion}

\subsection{Experimental Rationale and Design}

DNA repair of CPDs and 6-4 PPs in E. coli is limited to NER, except for CPD photoreversal under appropriate activation conditions. However, analyses of DNA repair pathways in certain viruses and other prokaryotic organisms reveal that alternative repair mechanisms, including BER and NIR are possible, but in E. coli, key enzymes are not present 
to initiate either pathway for the repair of UV-induced dipyrimidine photoproducts. To gain an understanding of how these alternative pathways may modulate cell survival in DNA repairproficient and-deficient E. coli, we implemented a strategy to activate the BER pathway for CPDs and the ring-fragmented purines, FapyAde and FapyGua, using cv-pdg. In parallel, NIR was activated for CPDs and 6-4 PPs by expression of $S$. pombe UVDE. Previous investigations have consistently revealed that expression of T4-pdg [30-32] or cv-pdg [24] in UVC-irradiated E. coli AB2480 (both NER- and HR-deficient), resulted in increased survival to levels approaching that observed with either the NER or HR single mutant, but not to WT levels. However, consequences of activating BER pathway using biologically relevant wavelengths of UV light have not been studied. The alteration of cellular responses via activation of the NIR pathway in E. coli has not been addressed under any conditions of UV exposure. Such analyses have the potential to distinguish the relative importance of repair of oxidatively-induced DNA damage versus 6-4 PP repair in a bacterial context.

\subsection{FapyGua and FapyAde are not substrates for UVDE}

UVDE has been previously shown to incise DNAs containing CPDs, 6-4 PPs, abasic sites, small loops, mismatches and other DNA lesions [34-36]. To understand the contribution of UVDE-initiated repair to survival following UVB and SSL exposures, we examined the ability of UVDE to release FapyGua or FapyAde from $\gamma$-irradiated DNA. Since we had previously demonstrated using GC-MS methods, that cv-pdg efficiently excised these ring-fragmented purines [26], we exploited these activities to determine whether UVDE could incise DNA at these sites. To serve as a control for release of FapyGua or FapyAde, purified cv-pdg was incubated with $\gamma$-irradiated calf thymus DNA in buffers containing either no metal ions or $\mathrm{MgCl}_{2}$ and $\mathrm{MnCl}_{2}$. As anticipated, cv-pdg released FapyAde and FapyGua (Fig. 1, Panels A and B, respectively) in the absence or presence of $\mathrm{MgCl}_{2}$ and $\mathrm{MnCl}_{2}$. Identical reactions were also performed using UVDE that exhibits maximum activity in the presence of these metal ions. Since UVDE has been previously characterized as an endonuclease, not a glycosylase, and this 
assay measures free base release, it was not surprising that levels of FapyAde and FapyGua were indistinguishable from the no enzyme controls following incubation in either the presence or absence of the divalent metal ions (Fig. 1, Panels A and B). To determine if UVDE had incised the DNA at fragmented purines, DNA was incubated with UVDE for $1 \mathrm{~h}$ prior to the addition of cV-pdg. It was reasoned that if UVDE incised DNA at sites containing FapyAde or FapyGua, these would not be further cleavable as result of subsequent addition of cv-pdg. However, if FapyAde and FapyGua remained intact, subsequent reaction with cv-pdg should release equivalent amounts of these base adducts as compared to incubation with cv-pdg alone. Data generated from the sequential addition of UVDE for $1 \mathrm{~h}$ and $\mathrm{cv}$-pdg for an additional $1 \mathrm{~h}$ (UVDE + cv-pdg) showed that the same quantity of both lesions was released, demonstrating that UVDE does not incise DNA at either FapyAde or FapyGua sites (Fig. 1, Panels A and B, respectively). Thus, although cv-pdg and UVDE can both initiate repair of CPDs, these enzymes have distinct substrate specificities. While UVDE incises DNA containing 6-4 PPs, cv-pdg catalyzes removal of FapyAde and FapyGua.

\subsection{Modulation of E. coli survival following UVB exposure by expression of cv-pdg or UVDE}

Having established the substrate specificities of cV-pdg and UVDE, we determined if expression of these enzymes in repair-proficient or -deficient $E$. coli could modulate cell survival following exposure to UVB. Plasmids were constructed that positioned the genes encoding cVpdg and UVDE downstream of a T7-RNA polymerase promoter that produces low, steady-state levels of each of these enzymes. These un-induced levels of expression of cv-pdg and UVDE were sufficient to exert a robust biological response in NER-deficient $E$. coli cells following challenge to irradiation as determined by UVB (and SSL) gradient dose responses, but did not cause noticeable toxicity under normal growth conditions (data not shown).

To quantify the effect of cV-pdg and UVDE on survival of $E$. coli following UVB irradiation, serial dilutions of WT and NER-deficient $\left(U v r A^{-}\right)$cells were challenged with increasing doses of UVB and colony formation was measured (Fig. 2, Panels A and B, respectively). As expected, 
the NER-deficient strain was much more sensitive than WT to UVB exposure, with equivalent doses required to reduce survival to $37 \%$ being $\sim 8$ and $79 \mathrm{~J} / \mathrm{m}^{2}$, respectively. Expression of either cV-pdg or UVDE did not enhance survival of WT E. coli at any of the doses examined (Panel A). In contrast, both cv-pdg and UVDE significantly improved survival of the NERdeficient cells at all doses $\left(p=0.00000062\right.$ and 0.00062 at $10 \mathrm{~J} / \mathrm{m}^{2}, 0.0000014$ and 0.0000019 at $20 \mathrm{~J} / \mathrm{m}^{2}$, and 0.00038 and 0.00020 at $30 \mathrm{~J} / \mathrm{m}^{2}$, respectively). Enhancement conferred by cvpdg was greater than that measured for ectopic expression of UVDE $\left(p=0.010\right.$ at $10 \mathrm{~J} / \mathrm{m}^{2}$, 0.00013 at $20 \mathrm{~J} / \mathrm{m}^{2}$, and 0.0033 at $30 \mathrm{~J} / \mathrm{m}^{2}$ ). Since this phenomenon was observed at all three doses tested, it is unlikely to be explained by possible differences in either the expression levels of active enzymes or availabilities of downstream activities to complete BER or NIR. An alternative explanation could be that there may only be a minor contribution of 6-4 PPs to the cytotoxic effects of UVB in E. coli and that enhanced repair of ring-fragmented purines by cVpdg may be more important for tolerating UVB. This assumption would be consistent with the observations that in both isolated and cellular DNAs exposed to UVB, 6-4 PPs are generated at a much lower frequency than CPDs (reviewed in [1]). Ectopic expression of either enzyme did not fully restore survival of the UvrA-deficient cells to that of WT, with equivalent doses required to reduce survival to $37 \%$ being 16 and $28 \mathrm{~J} / \mathrm{m}^{2}$ in UvrA-deficient cells expressing UVDE and cvpdg respectively, relative to $79 \mathrm{~J} / \mathrm{m}^{2}$ in WT. These data suggest that the initiation of alternative pathways such as BER and NIR, could not fully substitute for the intact NER system of E. coli.

The observation that there was no enhanced survival following UVB exposure in WT cells containing plasmids for the expression of cv-pdg or UVDE, raised the concern that NER may compete with BER and NIR for either common DNA substrates, shared downstream activities, or other factors that limit repair. Thus, experiments were designed to examine whether it was possible to increase survival by expressing these enzymes in any other NER-proficient strain. Since HR-deficient cells are also known to be UVB hypersensitive $[42,43]$, E. coli lacking functional RecA, an essential HR component [11], was used to address this question. RecA- 
deficient cells were transformed with the same set of three plasmids and following selection, were challenged with increasing doses of UVB (Fig. 2, Panel C). These data showed that even though the NER pathway was intact, activation of either cV-pdg- or UVDE-initiated repair enhanced survival $\left(p=0.11\right.$ and 0.067 at $5 \mathrm{~J} / \mathrm{m}^{2}, 0.031$ and 0.0085 at $10 \mathrm{~J} / \mathrm{m}^{2}$, and 0.036 and 0.061 at $20 \mathrm{~J} / \mathrm{m}^{2}$, respectively). Also, consistently observed at all doses, but not statistically significant, there was a trend toward greater enhancements in survival in cells expressing cvpdg versus UVDE. These data reveal that in the absence of HR, survival of $E$. coli as conferred by NER can be augmented by activation of either BER or NIR.

\subsection{Modulation of E. coli survival following SSL exposure by expression of cv-pdg or UVDE}

While the investigation described above focused on the biological effects of cellular response to a very narrow range of UVB wavelengths, terrestrial exposure to UV irradiation is significantly more complex and can be modelled by SSL. Our SSL experimental system generates $\sim 4 \%$ UVB relative to the total of UVA and UVB. To determine if expression of either cV-pdg or UVDE would modulate survival of WT following SSL exposure, cultures were exposed to increasing durations of SSL and the colony-forming abilities were measured (Fig. 3). Although survival of WT E. coli expressing UVDE was not significantly different from control at the 8-min and 16-min exposures, it was significantly enhanced at the 24 -min exposure $(p=0.034)$. The effect of expression of cv-pdg was even greater, with significant differences observed at the 16min and 24-min exposures ( $p=0.025$ and 0.020 , respectively) (Fig. 3). Similar analyses were carried out in UvrA-deficient cells, with the trends of enhanced survival observed for cells complemented with either cv-pdg or UVDE at the 24 min exposure (data not shown). Thus, the spectra of SSL-induced DNA damage include a fraction of cytotoxic lesions that can be repaired via initiation of BER or NIR. Since enhancement of survival by expression of cv-pdg or UVDE was observed in WT cells (Fig. 3), the cytotoxic SSL-induced lesions that represent substrates for these repair enzymes, are inefficiently, if not at all, recognized by NER. This conclusion agrees with prior studies demonstrating that the major component of solar UV, UVA, causes 
oxidative stress conditions [4-6] and that many common oxidatively-induced DNA lesions represent poor substrates for NER [9].

\section{Conclusions}

The data presented herein demonstrate the efficacy of enhancing the DNA repair capacity in WT and repair-deficient E. coli cells following exposures to UVB irradiation and to a limited extent, SSL. Extrapolation of these prokaryotic data suggest that mammalian cells might also benefit from enhanced DNA repair capacity of CPDs, 6-4 PPs, or oxidatively-induced DNA damage caused by UV exposure.

\section{Conflict of interest}

The authors declare no conflict of interest.

\section{Acknowledgements}

We wish to thank Dr. Paul W. Doetsch, Emory University for the expression plasmid for the truncated form of UVDE, Drs. Vladimir Vartanian, Nichole Owen, and Anuradha Kumari for laboratory and figure presentation assistance, and Dr. Amanda McCullough for guidance and helpful consultations throughout these investigations. Certain commercial equipment or materials are identified in this paper to specify adequately the experimental procedure. Such identification does not imply recommendation or endorsement by the National Institute of Standards and Technology, nor does it imply that the materials or equipment identified are necessarily the best available for the purpose. 


\section{References}

1. J. Cadet, A. Grand, T. Douki, Solar UV radiation-induced DNA Bipyrimidine photoproducts: formation and mechanistic insights, Top Curr Chem 356 (2015) 249-275.

2. D.E. Brash, UV signature mutations, Photochem Photobiol 91 (2015) 15-26.

3. G.M. Halliday, D.L. Damian, S. Rana, S.N. Byrne, The suppressive effects of ultraviolet radiation on immunity in the skin and internal organs: implications for autoimmunity, $\mathrm{J}$ Dermatol Sci 66 (2012) 176-182.

4. J. Cadet, T. Douki, J.L. Ravanat, Oxidatively generated damage to cellular DNA by UVB and UVA radiation, Photochem Photobiol 91 (2015) 140-155.

5. P. Karran, R. Brem, Protein oxidation, UVA and human DNA repair, DNA Repair (Amst) 44 (2016) 178-185.

6. E. Kvam, R.M. Tyrrell, Induction of oxidative DNA base damage in human skin cells by UV and near visible radiation, Carcinogenesis 18 (1997) 2379-2384.

7. D.X. Nghiem, N. Kazimi, G. Clydesdale, H.N. Ananthaswamy, M.L. Kripke, S.E. Ullrich, Ultraviolet a radiation suppresses an established immune response: implications for sunscreen design, J Invest Dermatol 117 (2001) 1193-1199.

8. D.X. Nghiem, N. Kazimi, D.L. Mitchell, A.A. Vink, H.N. Ananthaswamy, M.L. Kripke, S.E. Ullrich, Mechanisms underlying the suppression of established immune responses by ultraviolet radiation, J Invest Dermatol 119 (2002) 600-608.

9. J.J. Truglio, D.L. Croteau, B. Van Houten, C. Kisker, Prokaryotic nucleotide excision repair: the UvrABC system, Chem Rev 106 (2006) 233-252.

10. A. Sancar, Mechanisms of DNA repair by photolyase and excision nuclease (Nobel Lecture), Angew Chem Int Ed Engl 55 (2016) 8502-8527.

11. R.P. Fuchs, Tolerance of lesions in E. coli: Chronological competition between translesion synthesis and damage avoidance, DNA Repair (Amst) 44 (2016) 51-58.

12. R.S. Lloyd, Base excision repair of cyclobutane pyrimidine dimers, Mutat Res 408 (1998) 159-170.

13. R.S. Lloyd, The initiation of DNA base excision repair of dipyrimidine photoproducts, Prog Nucleic Acid Res Mol Biol 62 (1999) 155-175.

14. M.L. Dodson, R.D. Schrock 3rd, R.S. Lloyd, Evidence for an imino intermediate in the T4 endonuclease $V$ reaction, Biochemistry 32 (1993) 8284-8290. 
15. R.D. Schrock 3rd, R.S. Lloyd, Reductive methylation of the amino terminus of endonuclease $\mathrm{V}$ eradicates catalytic activities. Evidence for an essential role of the amino terminus in the chemical mechanisms of catalysis, J Biol Chem 266 (1991) 17631-17639.

16. R.D. Schrock 3rd, R.S. Lloyd, Site-directed mutagenesis of the $\mathrm{NH}_{2}$ terminus of $\mathrm{T} 4$ endonuclease $\mathrm{V}$, The position of the alpha $\mathrm{NH}_{2}$ moiety affects catalytic activity, J Biol Chem 268 (1993) 880-886.

17. E.C. Friedberg, J.J. King, Endonucleolytic cleavage of UV-irradiated DNA controlled by the $\mathrm{V}+$ gene in phage T4, Biochem Biophys Res Commun 37 (1969) 646-651.

18. Z. Lu, Y. Li, Y. Zhang, G.F. Kutish, D.L. Rock, J.L. Van Etten, Analysis of $45 \mathrm{~kb}$ of DNA located at the left end of the chlorella virus PBCV-1 genome, Virology 206 (1995) 339352.

19. E.S. Miller, J.F. Heidelberg, J.A. Eisen, W.C. Nelson, A.S. Durkin, A. Ciecko, T.V. Feldblyum, O. White, I.T. Paulsen, W.C. Nierman, J. Lee, B. Szczypinski, C.M. Fraser, Complete genome sequence of the broad-host-range vibriophage KVP40: comparative genomics of a T4-related bacteriophage, J Bacteriol 185 (2003) 5220-5233.

20. F. Repoila, F. Tetart, J.Y. Bouet, H.M. Krisch, Genomic polymorphism in the T-even bacteriophages, EMBO J 13 (1994) 4181-4192.

21. V.G. DelVecchio, V. Kapatral, R.J. Redkar, G. Patra, C. Mujer, T. Los, N. Ivanova, I. Anderson, A. Bhattacharyya, A. Lykidis, G. Reznik, L. Jablonski, N. Larsen, M. D'Souza, A. Bernal, M. Mazur, E. Goltsman, E. Selkov, P.H. Elzer, S. Hagius, D. O'Callaghan, J.J. Letesson, R. Haselkorn, N. Kyrpides, R. Overbeek, The genome sequence of the facultative intracellular pathogen Brucella melitensis, Proc Natl Acad Sci U S A 99 (2002) 443-448.

22. A. Dufresne, M. Salanoubat, F. Partensky, F. Artiguenave, I.M. Axmann, V. Barbe, S. Duprat, M.Y. Galperin, E.V. Koonin, F. Le Gall, K.S. Makarova, M. Ostrowski, S. Oztas, C. Robert, I.B. Rogozin, D.J. Scanlan, N. Tandeau de Marsac, J. Weissenbach, P. Wincker, Y.I. Wolf, W.R. Hess, Genome sequence of the cyanobacterium Prochlorococcus marinus SS120, a nearly minimal oxyphototrophic genome, Proc Natl Acad Sci U S A 100 (2003) 10020-10025.

23. B.J. May, Q. Zhang, L.L. Li, M.L. Paustian, T.S. Whittam, V. Kapur, Complete genomic sequence of Pasteurella multocida, Pm70, Proc Natl Acad Sci U S A 98 (2001) 34603465.

24. J.F. Garvish, R.S. Lloyd, The catalytic mechanism of a pyrimidine dimer-specific glycosylase (pdg)/abasic lyase, Chlorella virus-pdg, J Biol Chem 274 (1999) 9786-9794. 
25. J.F. Garvish, R.S. Lloyd, Active-site determination of a pyrimidine dimer glycosylase, J Mol Biol 295 (2000) 479-488.

26. P. Jaruga, R. Jabil, A.K. McCullough, H. Rodriguez, M. Dizdaroglu, R.S. Lloyd, Chlorella virus pyrimidine dimer glycosylase excises ultraviolet radiation- and hydroxyl radicalinduced products 4,6-diamino-5-formamidopyrimidine and 2,6-diamino-4-hydroxy-5formamidopyrimidine from DNA, Photochem Photobiol 75 (2002) 85-91.

27. A.K. McCullough, M.T. Romberg, S. Nyaga, Y. Wei, T.G. Wood, J.S. Taylor, J.L. Van Etten, M.L. Dodson, R.S. Lloyd, Characterization of a novel cis-syn and trans-syn-II pyrimidine dimer glycosylase/AP lyase from a eukaryotic algal virus, Paramecium bursaria chlorella virus-1, J Biol Chem 273 (1998) 13136-13142.

28. M.J. Calkins, V. Vartanian, N. Owen, G. Kirkali, P. Jaruga, M. Dizdaroglu, A.K. McCullough, R.S. Lloyd, Enhanced sensitivity of Neil1 $1^{-/-}$mice to chronic UVB exposure, DNA Repair (Amst) 48 (2016) 43-50.

29. P.W. Doetsch, T.H. Zasatawny, A.M. Martin, M. Dizdaroglu, M. Monomeric base damage products from adenine, guanine, and thymine induced by exposure of DNA to ultraviolet radiation, Biochemistry 34 (1995) 737-742.

30. D.R. Dowd, R.S. Lloyd, Biological consequences of a reduction in the non-target DNA scanning capacity of a DNA repair enzyme, J Mol Biol 208 (1989) 701-707.

31. C. Nickell, M.A. Prince, R.S. Lloyd, Consequences of molecular engineering enhanced DNA binding in a DNA repair enzyme, Biochemistry 31 (1992) 4189-4198.

32. A. Recinos 3rd, R.S. Lloyd, Site-directed mutagenesis of the T4 endonuclease V gene: role of lysine-130, Biochemistry 27 (1988) 1832-1838.

33. A. Yasui, Alternative excision repair pathways, Cold Spring Harb Perspect Biol 5 (2013) doi: $10.1101 /$ cshperspect.a012617.

34. A.M. Avery, B. Kaur, J.S. Taylor, J.A. Mello, J.M. Essigmann, P.W. Doetsch, Substrate specificity of ultraviolet DNA endonuclease (UVDE/Uve1p) from Schizosaccharomyces pombe, Nucleic Acids Res 27 (1999) 2256-2264.

35. B. Kaur, A.M. Avery, P.W. Doetsch, Expression, purification, and characterization of ultraviolet DNA endonuclease from Schizosaccharomyces pombe, Biochemistry 37 (1998) 11599-11604.

36. B. Kaur, P.W. Doetsch, Ultraviolet damage endonuclease (Uve1p): a structure and strand-specific DNA endonuclease, Biochemistry 39 (2000) 5788-5796. 
37. J.L. Johnson, B.C. Lowell, O.P. Ryabinina, R.S. Lloyd, A.K. McCullough, TAT-mediated delivery of a DNA repair enzyme to skin cells rapidly initiates repair of UV-induced DNA damage, J Invest Dermatol 131 (2011) 753-761.

38. P. Jaruga, E. Coskun, K. Kimbrough, A. Jacob, W.E. Johnson, M. Dizdaroglu, Biomarkers of oxidatively induced DNA damage in dreissenid mussels: A genotoxicity assessment tool for the Laurentian Great Lakes, Environ Toxicol 32 (2017) 2144-2153.

39. M. Dizdaroglu, The use of capillary gas chromatography-mass spectrometry for identification of radiation-induced DNA base damage and DNA base-amino acid crosslinks, J Chromatogr 295 (1984) 103-121.

40. M. Dizdaroglu, Application of capillary gas chromatography-mass spectrometry to chemical characterization of radiation-induced base damage of DNA: implications for assessing DNA repair processes, Anal Biochem 144 (1985) 593-603.

41. M. Dizdaroglu, Quantitative determination of oxidative base damage in DNA by stable isotope-dilution mass spectrometry, FEBS Lett 315 (1993) 1-6.

42. S.P. Kidambi, M.G. Booth, T.A. Kokjohn, R.V. Miller, RecA-dependence of the response of Pseudomonas aeruginosa to UVA and UVB irradiation, Microbiology 142 (Pt 4) (1996) 1033-1040.

43. A.G. Miguel, R.M. Tyrrell, Induction of oxygen-dependent lethal damage by monochromatic UVB $(313 \mathrm{~nm})$ radiation: strand breakage, repair and cell death, Carcinogenesis 4 (1983) 375-380. 


\section{Figure Legends}

Figure 1. Lack of excision of FapyAde and FapyGua by UVDE from $\gamma$-irradiated DNA. Calf thymus DNA in a $\mathrm{N}_{2} \mathrm{O}$-saturated phosphate buffer was irradiated with a 10 Gy dose of $\boldsymbol{\gamma}$-rays using a ${ }^{60} \mathrm{Co} \gamma$ ray-source, dialyzed, and $50 \mu \mathrm{g}$ aliquots incubated with cv-pdg alone, UVDE alone, or sequential addition of UVDE for $1 \mathrm{~h}$ followed by cV-pdg. The amounts of FapyAde (Panel A) and FapyGua (Panel B) released were measured by GC-MS/MS. The incision reactions were performed in the absence or presence of $\mathrm{MnCl}_{2}$ and $\mathrm{MgCl}_{2}$. Four independently prepared samples were used for each data point. The uncertainties are standard deviations.

\section{Figure 2. Survival of WT and repair-deficient $E$. coli strains that express enzymes to} activate BER and NIR pathways for UVB-induced DNA damage. WT (Panel A), UvrAdeficient (Panel B), and RecA-deficient (Panel C) E. coli that expressed cv-pdg (circles) or UVDE (squares) or contained an empty vector (triangles), were irradiated with increasing levels of UVB and colony forming ability measured. At least three independent experiments were used for each data point. The data are presented as means with standard errors. The $p$ values were calculated using two-tailed Student's $t$-test.

\section{Figure 3. Survival of WT E. coli that express enzymes to activate BER and NIR pathways} for SSL-induced DNA damage. WT E. coli that expressed cv-pdg (circles) or UVDE (squares) or contained an empty vector (triangles), were irradiated with increasing levels of SSL and colony forming ability measured. At least three independent experiments were used for each data point. The data are presented as means with standard errors. The $p$ values were calculated using two-tailed Student's $t$-test. 
Table 1. E. coli strains used in this study

\begin{tabular}{|c|c|}
\hline Name & Genotype \\
\hline AB2500 & $\begin{array}{l}\mathrm{F}^{-}, \text {thr-1, araC14, leuB6(Am), } \Delta(\text { gpt-proA)62, lacY1, tsx-33, qsr'-0, } \\
\text { glnX44(AS), galK2(Oc), } \lambda^{-} \text {, hisG4(Oc), thyA15, rpsL31(strR), xylA5, mtl-1, } \\
\operatorname{argE3(Oc),~thiE1,~uvrA6,~deoB12~}\end{array}$ \\
\hline AB1157 & $\begin{array}{l}\mathrm{F}^{-}, \text {thr-1, araC14, leuB6(Am), } \Delta \text { (gpt-proA)62, lacY1, tsx-33, qsr'-0, } \\
\text { glnX44(AS), galK2(Oc), } \lambda-\text {, Rac-0, hisG4(Oc), rfbC1, mgl-51, rpoS396(Am), } \\
\text { rpsL31(strR), kdgK51, xylA5, mtl-1, argE3(Oc), thiE1 }\end{array}$ \\
\hline AB2487 & $\begin{array}{l}\mathrm{F}^{-}, \text {thr-1, araC14, leuB6(Am), } \Delta \text { (gpt-proA)62, lacY1, tsx-33, qsr'-0, } \\
\text { glnX44(AS), galK2(Oc), } \lambda-, \text { Rac-0, hisG4(Oc), rfbC1, recA13, thyA16, } \\
\text { rpsL31(strR), kdgK51, xylA5, mtl-1, argE3(Oc), thiE1, deoB11 }\end{array}$ \\
\hline
\end{tabular}




\section{Figure 1}

A

\section{FapyAde}

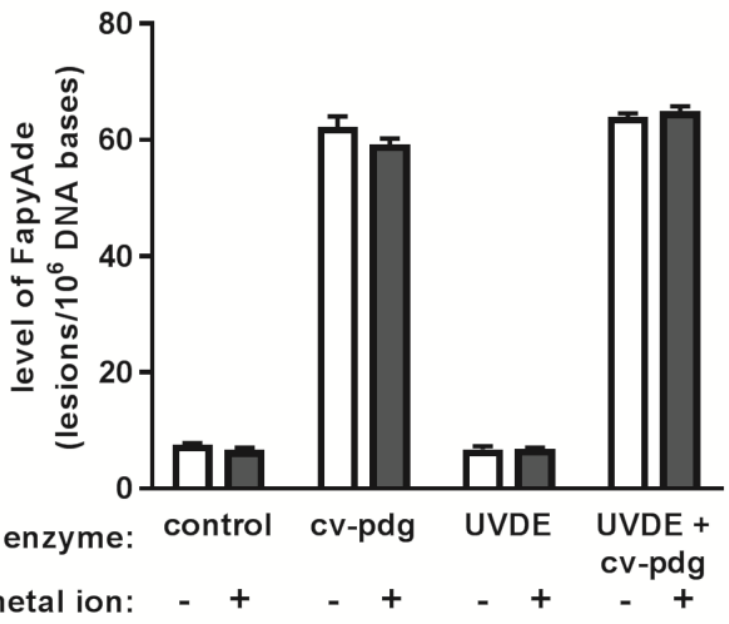

B

\section{FapyGua}

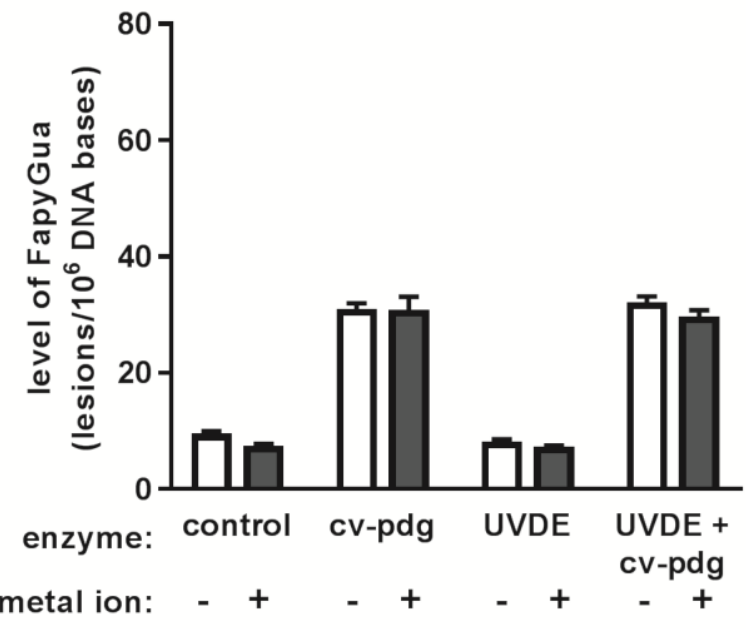




\section{Figure 2}
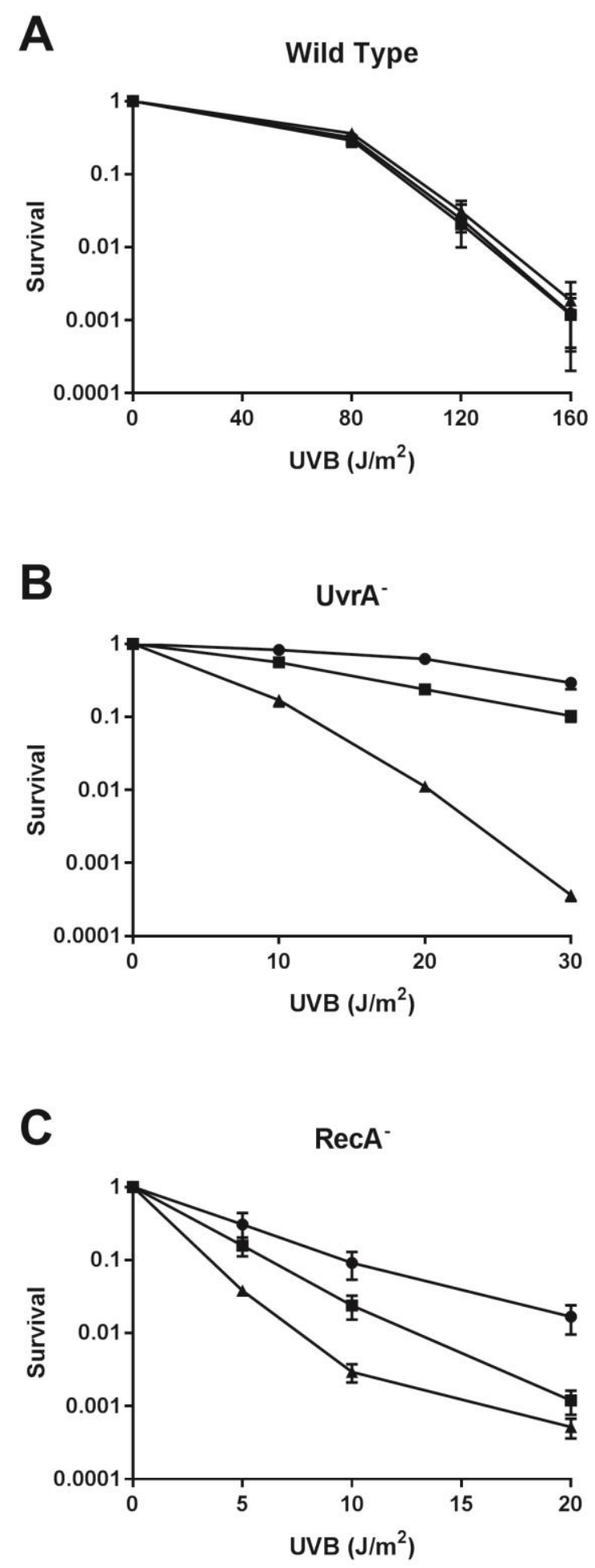


\section{Figure 3}

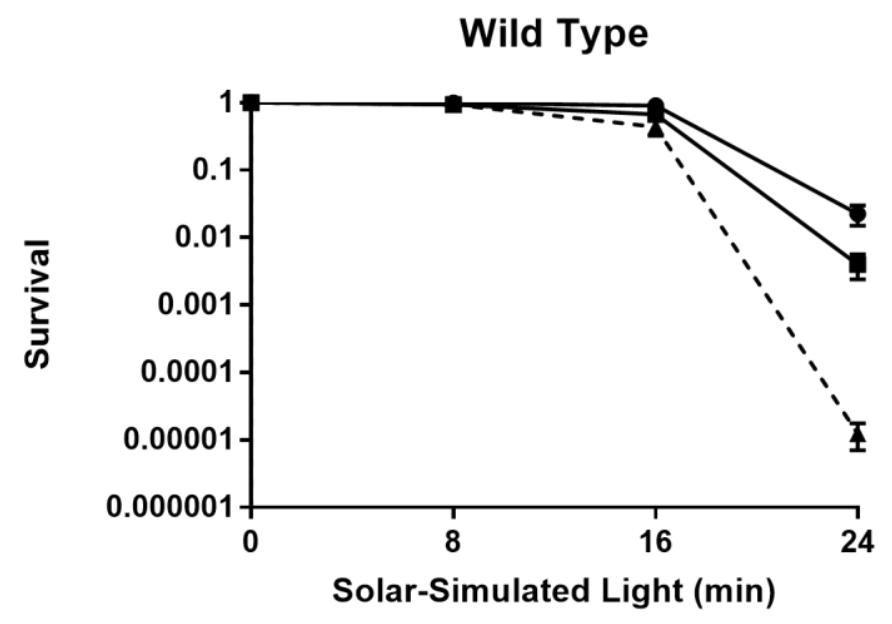

